\title{
Calibration of ionization chamber survey meter
}

\author{
Ali Kareem Kadhim ${ }^{1}$, Taiman Bin Kadni \\ ${ }^{1}$ Ministry of Science \& Technology, Iraq \\ ${ }^{2}$ SSDL, Malaysia Nuclear Agency \\ E-mail: alieinsteingh@yahoo.com
}

\begin{abstract}
Radiation measuring devices need to process calibration which lose their sensitivity and the extent of the response and the amount of stability under a changing conditions from time to time and this period depends on the nature and use of field in which used devices. A comparison study was done to a (451P) (ionization chamber survey meter) and this showed the variation of calibration factor in five different years. This study also displayed the concept of radiation instrument calibration and necessity of every year calibration of them.

In this project we used the five years calibration data for ionization chamber survey meter model Inspector (451P) to get that the values of Calibration Factor (CF) and Response (1/CF). The value deviation $(\triangle \%)$ of CFs for four years of calibration in comparison of CF for the year 2007 are very high and the device under research is not good to use in field and reliable because the ionization chamber is very sensitive to humidity and must calibrate a year or less, or during every two years and must maintain carefully to reduce the discarded effects to the measurements.
\end{abstract}

\section{Article info.} Received: Feb. 2016 Accepted: Mar. 2016 Published: Apr. 2016

Key words

Calibration factor, Ionization Chamber, Deviation.

$$
\begin{aligned}
& \text { معايرة جهاز القياس الإشعاعي (غرفة التأين) } \\
& \text { علي كريم كاظم 1، تايمان بن كادني2 }
\end{aligned}
$$

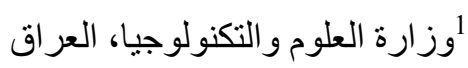

$$
\begin{aligned}
& \text { 2مختبرات التعبير الثانوي، الوكالة النووية الماليزية }
\end{aligned}
$$

الخلاصة

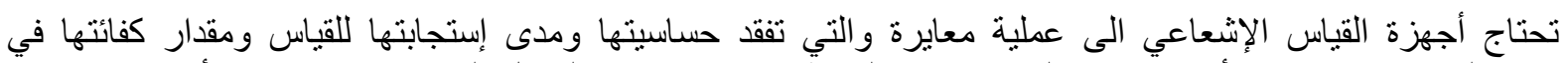

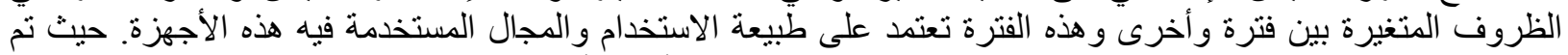

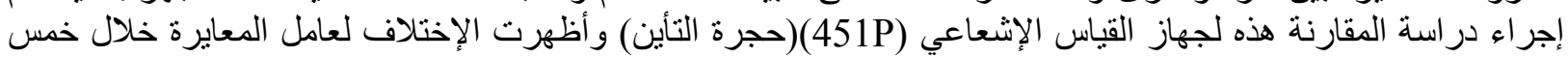

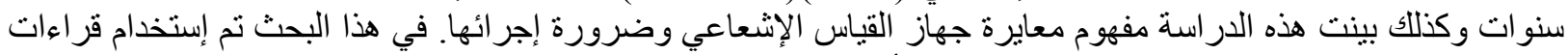

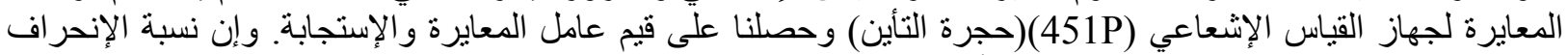

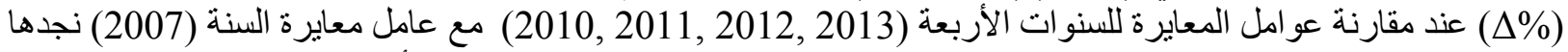

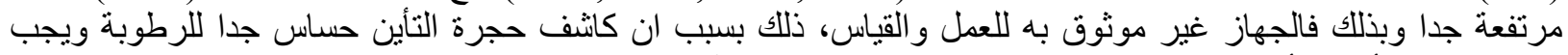
معايرته سنويا أو اقل أو خلال كل سنتين و المحافظة عليه للحد من الآثار السلبية المحتملة على القياسات.

\section{Introduction}

Radiation instruments used as survey monitors are either gas filled detectors or solid state detectors (e.g. scintillator or semiconductor detectors).
A gas filled detector is usually cylindrical in shape, with an outer wall and a central electrode well insulated from each other. The wall is usually made of tissue equivalent material for 
ionization chamber detectors and of brass or copper for other types of detector $[1,2]$.

The purposes of calibration are:

$>$ To determine calibration factors and response of $\mathrm{x}$ and gamma radiation of ionization chamber survey meters [3].

$>$ To determine of photon energy dependence of radiation survey meters.

$>$ Comparison of calibration factors and response using $\mathrm{x}$ and gamma radiation of same model of ionization chamber survey meter with different years $[3,4]$.

$>$ To ensure that an instrument is working properly and hence will be suitable for its intended monitoring purpose.

$>$ To adjust the instrument calibration, if possible, so that the overall measurement accuracy of the instrument is optimized.

\section{Calibration of survey meters}

Calibration is defined as the quantitative determination, under a controlled set of standard conditions, of the indication given by a radiation measuring instrument as a function of the value of the quantity the instrument is intended to measure.

In general, reference instruments do not directly indicate the appropriate dose equivalent quantity for calibrations or type tests. Instead, most frequently reference instruments are used to characterize the reference radiation fields by other measured such as fluence for neutron radiation and air kerma for photon radiation (Fig.1).

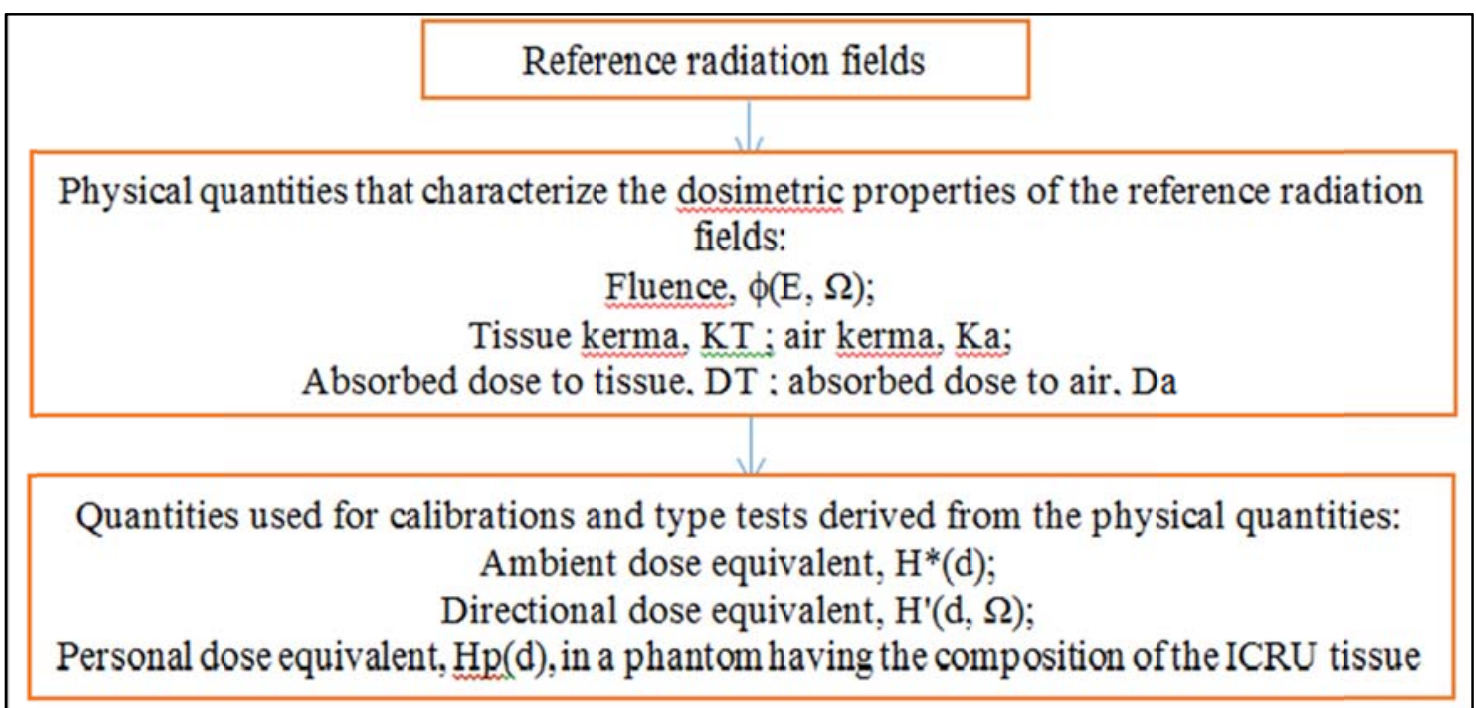

Fig.1: Reference radiation fields, physical quantities that characterize the dosimetric properties of the reference radiation fields, and quantities used for calibrations and type tests.

Protection level area survey meters must be calibrated against a reference instrument that is traceable (directly or indirectly) to a national standards laboratory $[4,5$, and 6$]$.

A reference instrument for $\gamma$ radiation is generally an ionization chamber with a measuring assembly. Reference instruments do not indicate directly the dose equivalent $H$ required for calibration of radiation protection monitoring instruments. Rather, they measure basic radiation quantities such as the air kerma in air for photon radiation, and the dose equivalent $H$ is then determined by using appropriate conversion coefficients $h$ :

$H=h C F_{\mathrm{R}} M_{\mathrm{R}}[3]$

Where $C_{R}$ is the calibration factor (e.g. in terms of air kerma in air or air kerma rate in air) of the reference chamber under reference conditions; 
$M_{R}$ is the reading of the reference instrument corrected for influence quantities.

A reference instrument is calibrated free in air for the range of reference radiation qualities (defined by the International Organization for Standardization (ISO)). The same reference qualities should be used for the calibration of radiation protection monitoring instruments.

Typically, calibration of survey meters in terms of the ambient dose equivalent $H^{*}(10)$ involves three steps:

- The air kerma in air is measured in a reference field, using a reference standard.

- The values of the conversion coefficient:

$\mathrm{h}_{\mathrm{H}^{*}}=\left[\mathrm{H}^{*}(10) /\left(\mathrm{K}_{\mathrm{air}}\right)_{\mathrm{air}}\right]$ [3].

are theoretically available. Using these data for the calibration beam quality, a reference instrument reading can be converted to $\mathrm{H}^{*}(10)$.

The survey monitor being calibrated is then placed at the calibration point and its reading $M$ is determined. The calibration factor in terms of the ambient dose equivalent $N_{H^{*}}$ for the survey monitor is determined from the equation

$\mathrm{CF}_{\mathrm{H}^{*}}=\mathrm{H}^{*}(10) / \mathrm{M}[3]$.

There are four methods of calibration of survey meter

- Method 1: calibration with a reference instrument without any monitor;

- Method 2: calibration with a reference instrument and with a monitor;

- Method 3: calibration by simultaneous irradiation of reference instrument and instrument under calibration;

- Method 4: calibration in a known radiation field [3, 7, and 8$]$.

In this project calibrations are performed either by the substitution method (Method 4, comparing the response of the instrument to be calibrated with that of a reference standard instrument) or simultaneous method (Method 3, both instrument to be calibrated and reference standard instrument are placed in the radiation beam at the same time and irradiated together). These methods are normally used when the radiation survey meters are calibrated for exposure to $\mathrm{x}$-ray beams. Calibration is performed in a known radiation field when the survey meters are calibrated against gamma, beta and neutron beams. For a radiation field in which the dose equivalent quantity $H$ of the field at the point of test is known, the calibration factor of an instrument $\mathrm{CF}$ is obtained by

$\mathrm{CF}=\mathrm{H} / \mathrm{M}$

where $C F$ is the calibration factor of the instrument under calibration (under reference conditions); $\mathrm{M}$ is the measured value of the instrument under calibration, corrected for reference conditions, i.e. multiplication by appropriate correction factors (e.g. differences in air density);

$\mathrm{H}$ is the conventional true value of the dose equivalent quantity to be measured.

\section{Materials}

Reference photon radiation selected from ISO Standard 4037-1 is used for calibration of radiation survey instruments and for the determination of their energy response. Standardization of ${ }^{137} \mathrm{Cs},{ }^{60} \mathrm{Co}$, and ${ }^{241} \mathrm{Am}$ gamma sources (protection level) at various distances using reference standard dosimeters are performed once a year with an accuracy of better than $\pm 2 \%$.

Calibration with beta radiation is performed using beta secondary standard sources whose absorbed dose rates at particular distances for each ${ }^{90} \mathrm{Sr} /{ }^{90} \mathrm{Y},{ }^{85} \mathrm{Kr}$ and ${ }^{147} \mathrm{Pm}$ sources have been determined by the PhysikalischTechnische Bundesanstalt (PTB), the national standard laboratory of 
Germany or by using PTW extrapolation ionization chamber.

In this work the following materials and instrument were used:

1. Pressurized ionization chamber survey meter model 451P-DE-SI-RYR.

2. Radiation sources.

a. Ceasium-137 (740GBq) and Cobalt60 (37GBq) sources from Gamma Calibrator model OB 85.

b. Ceasium-137 (7.4MBq - 7.4GBq) and Cobalt-60 (3.7MBq $-370 \mathrm{MBq})$ sources from Panoramic Gamma Irradiator model OB 34.

c. X-ray sources (Universal Bipolar Constant Potential X-ray Systems MG 325).

3. Working standard ionization chamber model PTW 32002 (1,000 cc).

4. Calibration bench. 5. CCTV.

6. Laser alignment. 7. Telescope.

8. PTW-Unidos electrometer.

9. Digital thermometer. 10. Barometer.

11. Hygrometer. 12. Area monitor.

13. Personal computer.

\section{Methods}

\section{Calibration using Cs-137 gamma radiation}

Calibration of the survey meter should be carried out on at least one point e.g. $50 \%$ of the full scale on each measuring range of the survey meter or in each decade for a survey meter with a logarithmic scale or with digital indication.

The conventional true value of exposure rate or dose rate at the point of test for survey meter are determined by using secondary standard ionization chamber.

The steps in the calibration procedures are:

$>$ Set the survey meter at appropriate exposure rate/dose rate range.

$>$ Place the survey meter in the calibrated source beam at appropriate distance from the sources. Ensure the survey meter is in the middle of the beam.
Expose the survey meter with radiation sources.

Record the meter reading displayed on the survey meter at least five times within a regular interval of time e.g. 10 seconds.

$>$ Calculate the average reading value and standard deviation.

Calculate the calibration factors.

$>$ Repeat these procedures for other exposure rate or dose rate range.

\section{Calibration using Co-60 gamma source}

The calibration should be carried out in the $50 \%$ of the full scale at one Measuring range. Repeat same procedures that followed with Cs-137 $[3,4]$.

\section{Calibration using $X$ - ray machine $(60 \mathrm{kV}-250 \mathrm{kV})$}

The calibration should be carried out in the $50 \%$ of the full scale at one measuring range.

$>$ Set the survey meter at low exposure rate/dose rate range.

$>$ Place the survey meter in the calibrated source beam at appropriate distance from the sources. The calibrated x-ray beam is determined by using working standard ionization chamber model PTW 32002, which has been calibrated against secondary standard ionization chamber. The survey meter and the ionization chamber must be the same distance from the X-ray source. First the working standard ionization chamber is used to determine the exposure rate or dose rate and then the reading of survey meter is determined.

$>$ Expose the working ionization chamber with $60 \mathrm{kV}$ X-ray with appropriate current $(\mathrm{mA})$. Determine the exposure rate or dose rate.

$>$ Expose the survey meter with $60 \mathrm{kV}$ X-ray.

$>$ Record the meter reading displayed on the survey meter at least five times 
within a regular interval of time e.g. 10 seconds.

Calculate the average reading value and standard deviation.

Calculate the calibration factors.

Repeat these procedures for other radiation qualities i.e. $100 \mathrm{kV}, 150 \mathrm{kV}$, $200 \mathrm{kV}$ and $250 \mathrm{kV}[3,4]$.
Results and discussion

1. Linearity Test using ${ }^{137} \mathrm{Cs}$

Linearity test must be performed previously before calibrate the ionization chamber survey meter, Linearity test is performed using gamma source ${ }^{137} \mathrm{Cs}$ given at Table 1 and is shown in Fig. 2.

Table 1: Linearity test using ${ }^{137} \mathrm{Cs}$.

\begin{tabular}{|c|c|c|c|c|c|c|c|c|}
\hline & \multirow{2}{*}{ Range } & \multicolumn{7}{|c|}{ Range correction factor } \\
\cline { 3 - 9 } & & $\mathbf{2 0 0 7}$ & $\mathbf{2 0 1 0}$ & $\mathbf{2 0 1 1}$ & $\mathbf{2 0 1 2}$ & $\mathbf{2 0 1 3}$ & Up border & Low border \\
\hline 1. & $0-5 \mu \mathrm{Sv} / \mathrm{h}$ & 0.93 & 1.056 & 0.934 & 0.96 & 0.78 & 1.2 & 0.8 \\
\hline 2. & $0-50 \mu \mathrm{Sv} / \mathrm{h}$ & 0.957 & 0.943 & 0.945 & 0.943 & 0.887 & 1.2 & 0.8 \\
\hline 3. & $0-500 \mu \mathrm{Sv} / \mathrm{h}$ & 0.954 & 0.995 & 1.002 & 1.006 & 0.973 & 1.2 & 0.8 \\
\hline 4. & $0-5 \mathrm{mSv} / \mathrm{h}$ & 1 & 1 & 1 & 1 & 1 & 1.2 & 0.8 \\
\hline 5. & $0-50 \mathrm{mSv} / \mathrm{h}$ & 0.984 & 1.025 & 1.025 & 1.042 & 0.957 & 1.2 & 0.8 \\
\hline
\end{tabular}

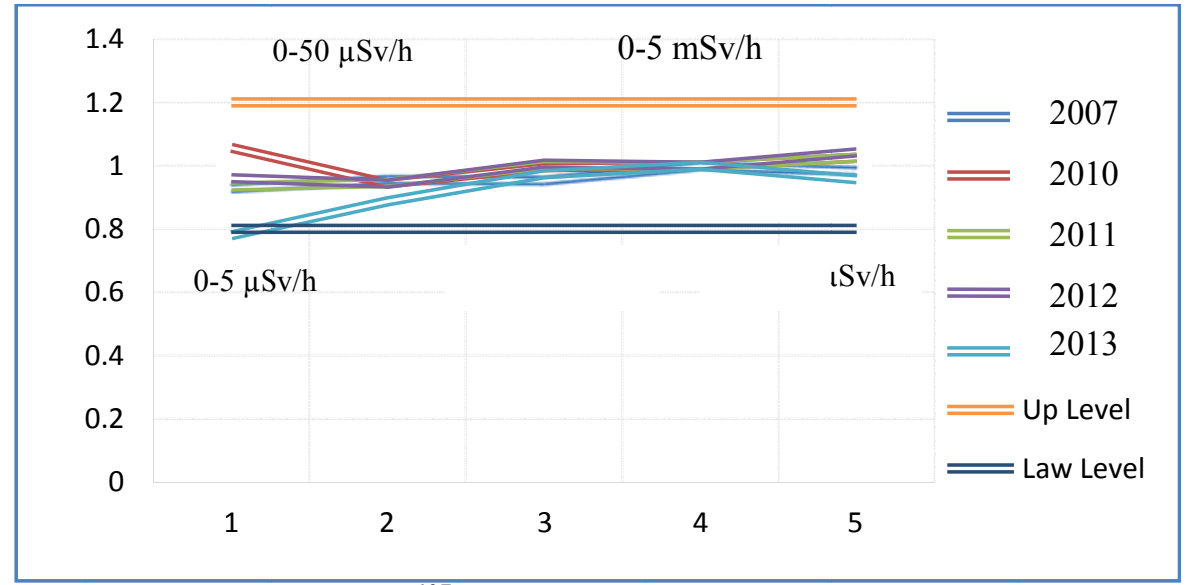

Fig.2: Linearity comparison, using ${ }^{137}$ Cs of ionisation chamber survey meter model $451 P$, for 5 different years.

To get true value of measurement using survey meter which has many scales besides using calibration factor also have to be corrected using range correction factor. This Fig.2 show about linearity of the ionisation chamber survey meter at 5 ranges, whereas linearity of survey meter acceptable when its deviation about \pm $20 \%$. From the graph, there is one of range correction factor (linearity) which has value less than 0.8 , so it is out of acceptable range.

\section{Calculate of the calibration factor and response}

Tables 2-6 shows the calculate calibration factors and response in 5 different years as shown in Fig. 3 and 4. 
Table 2: Calibration factors and response of 451P survey meter in 2007.

\begin{tabular}{|c|c|c|c|c|c|c|c|c|c|c|}
\hline \multirow{3}{*}{ R.Q } & \multirow[b]{3}{*}{$\begin{array}{c}\mathrm{C} \\
\mathrm{mA}\end{array}$} & \multirow[b]{3}{*}{$\begin{array}{c}\mathrm{E} \\
\mathrm{KeV}\end{array}$} & \multirow[b]{3}{*}{$\begin{array}{c}\text { CF.S } \\
\mathrm{mSv} / \mathrm{nC}\end{array}$} & \multirow{2}{*}{\multicolumn{3}{|c|}{ Readings }} & \multirow[b]{3}{*}{$\mathrm{CF}$} & \multirow[b]{3}{*}{$\begin{array}{c}\mathrm{R} \\
(1 / \mathrm{CF})\end{array}$} & \multirow[b]{3}{*}{$\begin{array}{c}\mathrm{HVL} \\
\mathrm{mm}\end{array}$} & \multirow[b]{3}{*}{$(\mathrm{T}),(\mathrm{P}),(\mathrm{H})$} \\
\hline & & & & & & & & & & \\
\hline & & & & $\begin{array}{c}\text { E.C } \\
\mathrm{nC} / \min \end{array}$ & $\begin{array}{c}\mathrm{S} . \mathrm{R} \\
\mathrm{mSv} / \mathrm{y}\end{array}$ & $\begin{array}{l}\text { SM.R } \\
\mathrm{mSv} / \mathrm{y}\end{array}$ & & & & \\
\hline \multirow[t]{2}{*}{ N-60 } & 2.88 & 47 & 0.0281 & 2.320 & 3.955 & 2.5 & 1.582 & 0.6321 & 0.24 & \multirow{9}{*}{$\begin{array}{c}\mathrm{T}: 21.76^{\circ} \mathrm{C} \\
\mathrm{P}: 1003.6 \mathrm{mbar} \\
\mathrm{H}: 66.36 \% \\
\mathrm{Ktp}: 1.016\end{array}$} \\
\hline & \multicolumn{9}{|c|}{ Additional filter : $4.0 \mathrm{~mm} \mathrm{Al}+0.62 \mathrm{~mm} \mathrm{Cu}$} & \\
\hline \multirow[t]{2}{*}{$\mathrm{N}-100$} & 8.75 & 84 & 0.0279 & 1.930 & 3.282 & 2.5 & 1.313 & 0.7616 & 1.14 & \\
\hline & \multicolumn{9}{|c|}{ Additional filter : $4.0 \mathrm{~mm} \mathrm{Al}+5.04 \mathrm{~mm} \mathrm{Cu}$} & \\
\hline \multirow[t]{2}{*}{$\mathrm{N}-150$} & 0.94 & 121 & 0.0283 & 1.857 & 3.198 & 2.5 & 1.279 & 0.7818 & 2.42 & \\
\hline & \multicolumn{9}{|c|}{ Additional filter : $4.0 \mathrm{~mm} \mathrm{Al}+2.5 \mathrm{~mm} \mathrm{Sn}$} & \\
\hline \multirow[t]{2}{*}{ N-200 } & 1.80 & 171 & 0.0284 & 1.796 & 3.114 & 2.5 & 1.246 & 0.8052 & 4.16 & \\
\hline & \multicolumn{9}{|c|}{ Additional filter : $4.0 \mathrm{~mm} \mathrm{Al}+2 \mathrm{~mm} \mathrm{Cu}+3 \mathrm{~mm} \mathrm{Sn}+1 \mathrm{~mm} \mathrm{~Pb}$} & \\
\hline \multirow[t]{2}{*}{$\mathrm{N}-250$} & 1.93 & 218 & 0.0290 & 1.809 & 3.193 & 2.5 & 1.277 & 0.7831 & 5.38 & \\
\hline & \multicolumn{9}{|c|}{ Additional filter : $4.0 \mathrm{~mm} \mathrm{Al}+2 \mathrm{~mm} \mathrm{Sn}+3 \mathrm{~mm} \mathrm{~Pb}$} & \\
\hline Cs-137 & - & 662 & - & - & 2.5 & 1.822 & 1.372 & 0.7288 & - & $\begin{array}{c}\mathrm{T}: 21.3^{\circ} \mathrm{C} \\
\mathrm{P}: 997.8 \mathrm{mbar} \\
\mathrm{H}: 51.63 \%\end{array}$ \\
\hline Co-60 & - & 1250 & - & - & 2.5 & 1.822 & 1.372 & 0.7288 & - & $\begin{array}{c}\mathrm{T}: 21.3^{\circ} \mathrm{C} \\
\mathrm{P}: 997.8 \mathrm{mbar} \\
\mathrm{H}: 51.63 \%\end{array}$ \\
\hline
\end{tabular}

Table 3: Calibration factors and response of 451P survey meter in 2010.

\begin{tabular}{|c|c|c|c|c|c|c|c|c|c|c|}
\hline \multirow[b]{2}{*}{ R.Q } & \multirow[b]{2}{*}{$\begin{array}{c}\mathrm{C} \\
\mathrm{mA}\end{array}$} & \multirow[b]{2}{*}{$\begin{array}{c}\mathrm{E} \\
\mathrm{KeV}\end{array}$} & \multirow[b]{2}{*}{$\begin{array}{c}\text { CF.S } \\
\mathrm{mSv} / \mathrm{nC}\end{array}$} & \multicolumn{3}{|c|}{ Readings } & \multirow[b]{2}{*}{$\mathrm{CF}$} & \multirow[b]{2}{*}{$\begin{array}{c}\mathrm{R} \\
(1 / \mathrm{CF})\end{array}$} & \multirow[b]{2}{*}{$\begin{array}{c}\mathrm{HVL} \\
\mathrm{mm}\end{array}$} & \multirow[b]{2}{*}{$(\mathrm{T}),(\mathrm{P}),(\mathrm{H})$} \\
\hline & & & & $\begin{array}{c}\text { E.C } \\
\mathrm{nC} / \mathrm{min}\end{array}$ & $\begin{array}{c}\text { S.R } \\
\mathrm{mSv} / \mathrm{y}\end{array}$ & $\begin{array}{l}\text { SM.R } \\
\text { mSv/y }\end{array}$ & & & & \\
\hline \multirow[t]{2}{*}{$\mathrm{N}-60$} & 3.05 & 47 & 0.0281 & 2.472 & 4.248 & 2.5 & 1.699 & 0.5886 & 0.24 & $\mathrm{~T}: 21.76^{\circ} \mathrm{C}$ \\
\hline & \multicolumn{9}{|c|}{ Additional filter : $4.0 \mathrm{~mm} \mathrm{Al}+0.62 \mathrm{~mm} \mathrm{Cu}$} & $P: 1003.6$ \\
\hline \multirow[t]{2}{*}{$\mathrm{N}-100$} & 9.30 & 84 & 0.0281 & 2.039 & 3.510 & 2.5 & 1.404 & 0.7122 & 1.08 & \\
\hline & \multicolumn{9}{|c|}{ Additional filter : $4.0 \mathrm{~mm} \mathrm{Al}+5.04 \mathrm{~mm} \mathrm{Cu}$} & H :66.36 \% \\
\hline \multirow[t]{2}{*}{$\mathrm{N}-150$} & 0.94 & 121 & 0.0284 & 1.916 & 3.424 & 2.5 & 1.370 & 0.7299 & 2.46 & Ktp :1.016 \\
\hline & \multicolumn{9}{|c|}{ Additional filter : $4.0 \mathrm{~mm} \mathrm{Al}+2.5 \mathrm{~mm} \mathrm{Sn}$} & \\
\hline \multirow[t]{2}{*}{$\mathrm{N}-200$} & 1.60 & 171 & 0.0286 & 1.916 & 3.356 & 2.5 & 1.342 & 0.7451 & 4.07 & \\
\hline & \multicolumn{9}{|c|}{ Additional filter : $4.0 \mathrm{~mm} \mathrm{Al}+2 \mathrm{~mm} \mathrm{Cu}+3 \mathrm{~mm} \mathrm{Sn}+1 \mathrm{~mm} \mathrm{~Pb}$} & \\
\hline \multirow[t]{2}{*}{$\mathrm{N}-250$} & 1.95 & 218 & 0.0288 & 1.9196 & 3.386 & 2.5 & 1.344 & 0.7440 & 5.32 & \\
\hline & \multicolumn{9}{|c|}{ Additional filter : $4.0 \mathrm{~mm} \mathrm{Al}+2 \mathrm{~mm} \mathrm{Sn}+3 \mathrm{~mm} \mathrm{~Pb}$} & \\
\hline Cs-137 & - & 662 & - & - & 2.5 & 1.471 & 1.372 & 0.6798 & - & $\begin{array}{c}\mathrm{T}: 21.3{ }^{\circ} \mathrm{C} \\
\mathrm{P}: 997.8 \mathrm{mbar} \\
\mathrm{H}: 51.63 \%\end{array}$ \\
\hline Сo-60 & - & 1250 & - & - & 2.5 & 1.400 & 1.372 & 0.7143 & - & $\begin{array}{c}\mathrm{T}: 21.3{ }^{\circ} \mathrm{C} \\
\mathrm{P}: 997.8 \mathrm{mbar} \\
\mathrm{H}: 51.63 \%\end{array}$ \\
\hline
\end{tabular}


Table 4: Calibration factors and response of 451P survey meter in 2011.

\begin{tabular}{|c|c|c|c|c|c|c|c|c|c|c|}
\hline \multirow[b]{2}{*}{ R.Q } & \multirow[b]{2}{*}{$\begin{array}{c}\mathrm{C} \\
\mathrm{mA}\end{array}$} & \multirow[b]{2}{*}{$\begin{array}{c}\mathrm{E} \\
\mathrm{KeV}\end{array}$} & \multirow[b]{2}{*}{$\begin{array}{c}\text { CF.S } \\
\mathrm{mSv} / \mathrm{nC}\end{array}$} & \multicolumn{3}{|c|}{ Readings } & \multirow[b]{2}{*}{$\mathrm{CF}$} & \multirow[b]{2}{*}{$\begin{array}{c}\mathrm{R} \\
(1 / \mathrm{CF})\end{array}$} & \multirow[b]{2}{*}{$\begin{array}{c}\mathrm{HVL} \\
\mathrm{mm}\end{array}$} & \multirow[b]{2}{*}{$(\mathrm{T}),(\mathrm{P}),(\mathrm{H})$} \\
\hline & & & & $\begin{array}{c}\text { E.C } \\
\mathrm{nC} / \mathrm{min}\end{array}$ & $\begin{array}{c}\mathrm{S} . \mathrm{R} \\
\mathrm{mSv} / \mathrm{y}\end{array}$ & $\begin{array}{l}\text { SM.R } \\
\text { mSv/y }\end{array}$ & & & & \\
\hline \multirow[t]{2}{*}{$\mathrm{N}-60$} & 4.78 & 47 & 0.0281 & 2.610 & 4.501 & 2.5 & 1.801 & 0.5552 & 0.24 & \multirow{9}{*}{$\begin{array}{c}\mathrm{T}: 22.5{ }^{\circ} \mathrm{C} \\
\mathrm{P}: 99.9 \mathrm{mbar} \\
\mathrm{H}: 48 \% \\
\mathrm{Ktp}: 1.023\end{array}$} \\
\hline & \multicolumn{9}{|c|}{ Additional filter : $4.0 \mathrm{~mm} \mathrm{Al}+0.62 \mathrm{~mm} \mathrm{Cu}$} & \\
\hline \multirow[t]{2}{*}{ N-100 } & 14.6 & 84 & 0.0281 & 2.190 & 3.774 & 2.5 & 1.510 & 0.662 & 1.08 & \\
\hline & \multicolumn{9}{|c|}{ Additional filter : $4.0 \mathrm{~mm} \mathrm{Al}+5.04 \mathrm{~mm} \mathrm{Cu}$} & \\
\hline \multirow[t]{2}{*}{$\mathrm{N}-150$} & 1.59 & 121 & 0.0285 & 2.0608 & 3.603 & 2.5 & 1.441 & 0.6993 & 2.46 & \\
\hline & \multicolumn{9}{|c|}{ Additional filter : $4.0 \mathrm{~mm} \mathrm{Al}+2.5 \mathrm{~mm} \mathrm{Sn}$} & \\
\hline \multirow[t]{2}{*}{ N-200 } & 2.51 & 171 & 0.0286 & 2.0489 & 3.359 & 2.5 & 1.340 & 0.6944 & 4.07 & \\
\hline & \multicolumn{9}{|c|}{ Additional filter : $4.0 \mathrm{~mm} \mathrm{Al}+2 \mathrm{~mm} \mathrm{Cu}+3 \mathrm{~mm} \mathrm{Sn}+1 \mathrm{~mm} \mathrm{~Pb}$} & \\
\hline \multirow[t]{2}{*}{$\mathrm{N}-250$} & 2.92 & 218 & 0.0289 & 2.0164 & 3.576 & 2.5 & 1.431 & 0.6988 & 5.32 & \\
\hline & \multicolumn{9}{|c|}{ Additional filter : $4.0 \mathrm{~mm} \mathrm{Al}+2 \mathrm{~mm} \mathrm{Sn}+3 \mathrm{~mm} \mathrm{~Pb}$} & \\
\hline Cs-137 & - & 662 & - & - & 2.5 & 1.636 & 1.528 & 0.6544 & - & $\begin{array}{c}\mathrm{T}: 21.5^{\circ} \mathrm{C} \\
\mathrm{P}: 996 \mathrm{mbar} \\
\mathrm{H}: 69.2 \%\end{array}$ \\
\hline Co-60 & - & 1250 & - & - & 2.5 & 1.752 & 1.427 & 0.7007 & - & $\begin{array}{c}\mathrm{T}: 23{ }^{\circ} \mathrm{C} \\
\mathrm{P}: 998 \mathrm{mbar} \\
\mathrm{H}: 50.5 \%\end{array}$ \\
\hline
\end{tabular}

Table 5: Calibration factors and response of 451P survey meter in 2012.

\begin{tabular}{|c|c|c|c|c|c|c|c|c|c|c|}
\hline \multirow[b]{2}{*}{ R.Q } & \multirow[b]{2}{*}{$\begin{array}{c}\mathrm{C} \\
\mathrm{mA}\end{array}$} & \multirow[b]{2}{*}{$\begin{array}{c}\mathrm{E} \\
\mathrm{KeV}\end{array}$} & \multirow[b]{2}{*}{$\begin{array}{c}\text { CF.S } \\
\mathrm{mSv} / \mathrm{nC}\end{array}$} & \multicolumn{3}{|c|}{ Readings } & \multirow[b]{2}{*}{$\mathrm{CF}$} & \multirow[b]{2}{*}{$\begin{array}{c}\mathrm{R} \\
(1 / \mathrm{CF})\end{array}$} & \multirow[b]{2}{*}{$\begin{array}{c}\mathrm{HVL} \\
\mathrm{mm}\end{array}$} & \multirow[b]{2}{*}{$(\mathrm{T}),(\mathrm{P}),(\mathrm{H})$} \\
\hline & & & & $\begin{array}{c}\text { E.C } \\
\mathrm{nC} / \mathrm{min}\end{array}$ & $\begin{array}{c}\mathrm{S} . \mathrm{R} \\
\mathrm{mSv} / \mathrm{y}\end{array}$ & $\begin{array}{l}\text { SM.R } \\
\mathrm{mSv} / \mathrm{y}\end{array}$ & & & & \\
\hline \multirow[t]{2}{*}{$\mathrm{N}-60$} & 4.0 & 47 & 0.0281 & 2.1944 & 3.763 & 2.0 & 1.882 & 0.5313 & 0.24 & \multirow{9}{*}{$\begin{array}{c}\mathrm{T}: 22{ }^{\circ} \mathrm{C} \\
\mathrm{P}: 1003 \mathrm{mbar} \\
\mathrm{H}: 40 \% \\
\mathrm{Ktp}: 1.017\end{array}$} \\
\hline & \multicolumn{9}{|c|}{ Additional filter : $4.0 \mathrm{~mm} \mathrm{Al}+0.62 \mathrm{~mm} \mathrm{Cu}$} & \\
\hline \multirow[t]{2}{*}{$\mathrm{N}-100$} & 11.8 & 84 & 0.0281 & 1.7804 & 3.051 & 2.0 & 1.525 & 0.6557 & 1.08 & \\
\hline & \multicolumn{9}{|c|}{ Additional filter : $4.0 \mathrm{~mm} \mathrm{Al}+5.04 \mathrm{~mm} \mathrm{Cu}$} & \\
\hline \multirow[t]{2}{*}{$\mathrm{N}-150$} & 1.30 & 121 & 0.0285 & 1.6864 & 2.932 & 2.0 & 1.466 & 0.6821 & 2.46 & \\
\hline & \multicolumn{9}{|c|}{ Additional filter : $4.0 \mathrm{~mm} \mathrm{Al}+2.5 \mathrm{~mm} \mathrm{Sn}$} & \\
\hline \multirow[t]{2}{*}{$\mathrm{N}-200$} & 2.10 & 171 & 0.0286 & 1.7082 & 2.983 & 2.0 & 1.492 & 0.6702 & 4.07 & \\
\hline & \multicolumn{9}{|c|}{ Additional filter : $4.0 \mathrm{~mm} \mathrm{Al}+2 \mathrm{~mm} \mathrm{Cu}+3 \mathrm{~mm} \mathrm{Sn}+1 \mathrm{~mm} \mathrm{~Pb}$} & \\
\hline \multirow[t]{2}{*}{$\mathrm{N}-250$} & 2.40 & 218 & 0.0289 & 1.7450 & 3.077 & 2.0 & 1.539 & 0.6498 & 5.32 & \\
\hline & \multicolumn{9}{|c|}{ Additional filter : $4.0 \mathrm{~mm} \mathrm{Al}+2 \mathrm{~mm} \mathrm{Sn}+3 \mathrm{~mm} \mathrm{~Pb}$} & \\
\hline Cs-137 & - & 662 & - & - & 2.5 & 1.598 & 1.564 & 0.6394 & - & $\begin{array}{c}\mathrm{T}: 22{ }^{\circ} \mathrm{C} \\
\mathrm{P}: 1001 \mathrm{mbar} \\
\mathrm{H}: 45 \%\end{array}$ \\
\hline Co-60 & - & 1250 & - & - & 2.5 & 1.752 & 1.427 & 0.7007 & - & $\begin{array}{c}\mathrm{T}: 21.3^{\circ} \mathrm{C} \\
\mathrm{P}: 1003 \mathrm{mbar} \\
\mathrm{H}: 50 \%\end{array}$ \\
\hline
\end{tabular}


Table 6: Calibration factors and response of 451P survey meter in 2013.

\begin{tabular}{|c|c|c|c|c|c|c|c|c|c|c|}
\hline \multirow[b]{2}{*}{ R.Q } & \multirow[b]{2}{*}{$\begin{array}{c}\mathrm{C} \\
\mathrm{mA}\end{array}$} & \multirow[b]{2}{*}{$\begin{array}{c}\mathrm{E} \\
\mathrm{KeV}\end{array}$} & \multirow[b]{2}{*}{$\begin{array}{c}\text { CF.S } \\
\mathrm{mSv} / \mathrm{nC}\end{array}$} & \multicolumn{3}{|c|}{ Readings } & \multirow[b]{2}{*}{$\mathrm{CF}$} & \multirow[b]{2}{*}{$\begin{array}{c}\mathrm{R} \\
(1 / \mathrm{CF})\end{array}$} & \multirow[b]{2}{*}{$\begin{array}{c}\mathrm{HVL} \\
\mathrm{mm}\end{array}$} & \multirow[b]{2}{*}{$(\mathrm{T}),(\mathrm{P}),(\mathrm{H})$} \\
\hline & & & & $\begin{array}{c}\text { E.C } \\
\mathrm{nC} / \mathrm{min}\end{array}$ & $\begin{array}{c}\mathrm{S} . \mathrm{R} \\
\mathrm{mSv} / \mathrm{y}\end{array}$ & $\begin{array}{l}\text { SM.R } \\
\mathrm{mSv} / \mathrm{y}\end{array}$ & & & & \\
\hline \multirow[t]{2}{*}{$\mathrm{N}-60$} & 3.40 & 47 & 0.0282 & 1.3614 & 2.35 & 2.5 & 0.941 & 1.0626 & 0.24 & \multirow{9}{*}{$\begin{array}{c}\mathrm{T}: 23{ }^{\circ} \mathrm{C} \\
\mathrm{P}: 1003 \mathrm{mbar} \\
\mathrm{H}: 57 \% \\
\mathrm{Ktp}: 1.021\end{array}$} \\
\hline & \multicolumn{9}{|c|}{ Additional filter : $4.0 \mathrm{~mm} \mathrm{Al}+0.62 \mathrm{~mm} \mathrm{Cu}$} & \\
\hline \multirow[t]{2}{*}{$\mathrm{N}-100$} & 9.0 & 84 & 0.0283 & 0.9298 & 1.61 & 1.918 & 0.842 & 1.1876 & 1.08 & \\
\hline & \multicolumn{9}{|c|}{ Additional filter : $4.0 \mathrm{~mm} \mathrm{Al}+5.04 \mathrm{~mm} \mathrm{Cu}$} & \\
\hline \multirow[t]{2}{*}{$\mathrm{N}-150$} & 1.53 & 121 & 0.0284 & 1.3790 & 2.40 & 2.5 & 0.961 & 1.0406 & 2.46 & \\
\hline & \multicolumn{9}{|c|}{ Additional filter : $4.0 \mathrm{~mm} \mathrm{Al}+2.5 \mathrm{~mm} \mathrm{Sn}$} & \\
\hline \multirow[t]{2}{*}{$\mathrm{N}-200$} & 2.55 & 171 & 0.0287 & 1.4538 & 2.56 & 2.5 & 1.023 & 0.9775 & 4.07 & \\
\hline & \multicolumn{9}{|c|}{ Additional filter : $4.0 \mathrm{~mm} \mathrm{Al}+2 \mathrm{~mm} \mathrm{Cu}+3 \mathrm{~mm} \mathrm{Sn}+1 \mathrm{~mm} \mathrm{~Pb}$} & \\
\hline \multirow[t]{2}{*}{$\mathrm{N}-250$} & 2.90 & 218 & 0.0289 & 1.4244 & 2.52 & 2.5 & 1.008 & 0.9920 & 5.32 & \\
\hline & \multicolumn{9}{|c|}{ Additional filter : $4.0 \mathrm{~mm} \mathrm{Al}+2 \mathrm{~mm} \mathrm{Sn}+3 \mathrm{~mm} \mathrm{~Pb}$} & \\
\hline Cs-137 & - & 662 & - & - & 2.5 & 2.20 & 1.136 & 0.8803 & - & $\begin{array}{c}\mathrm{T}: 23{ }^{\circ} \mathrm{C} \\
\mathrm{P}: 997 \mathrm{mbar} \\
\mathrm{H}: 74.1 \%\end{array}$ \\
\hline Co-60 & - & 1250 & - & - & 2.5 & 2.260 & 1.106 & 0.9041 & - & $\begin{array}{c}\mathrm{T}: 23{ }^{\circ} \mathrm{C} \\
\mathrm{P}: 997 \mathrm{mbar} \\
\mathrm{H}: 74.1 \%\end{array}$ \\
\hline
\end{tabular}

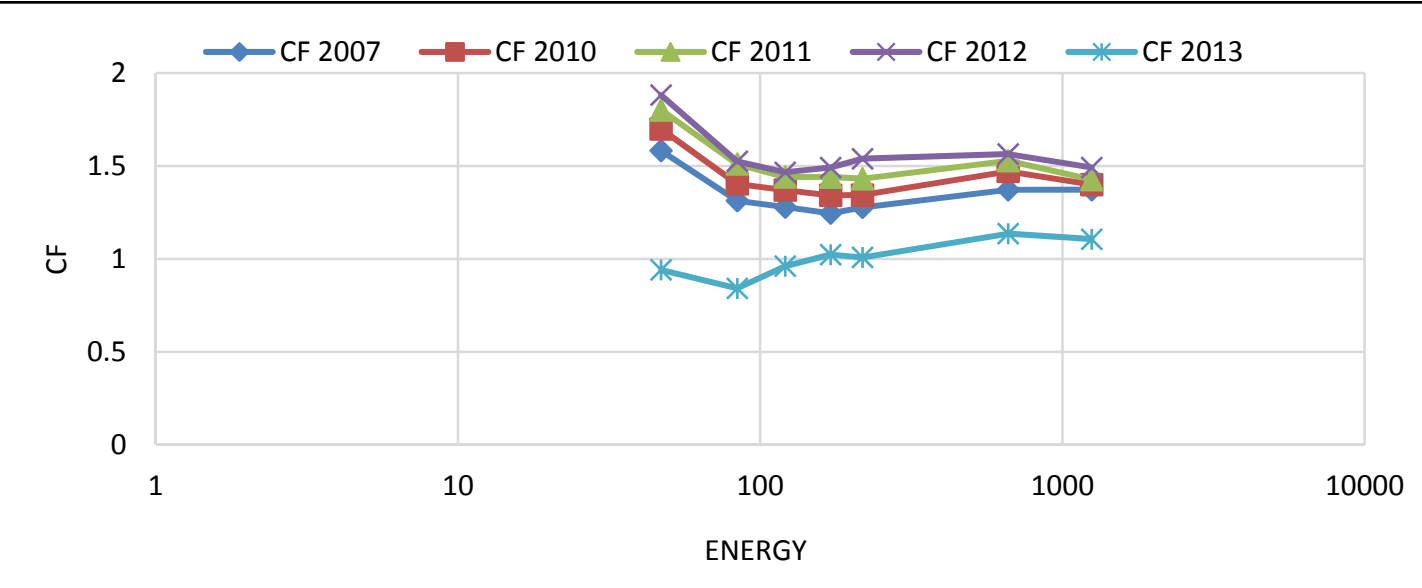

Fig.3: Calibration factors of 451P survey meter in 5 different years.

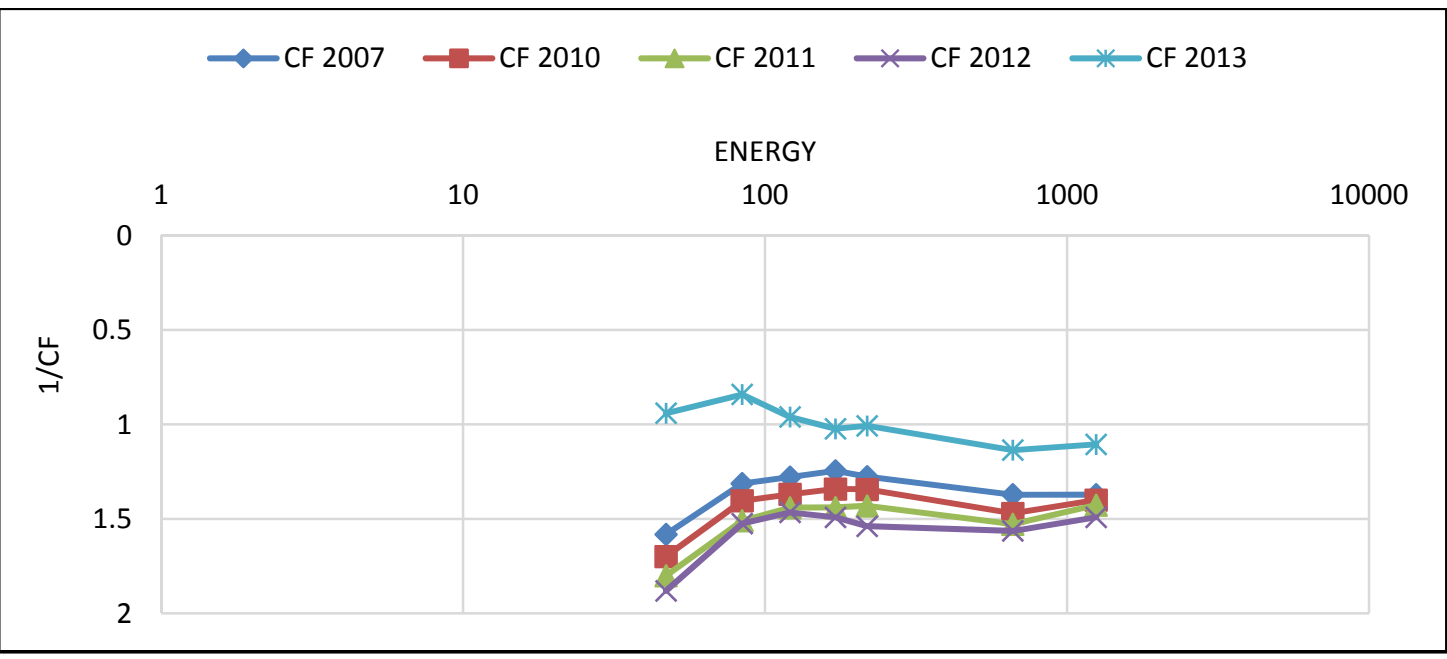

Fig.4: Response of $451 \mathrm{P}$ survey meter in 5 different years. 
Comparison of calibration factors between year 2007 and other year is shown in Table 7. As can be seen CFs are changing from year to other year.
Percentage deviation:

$$
\Delta \%=\frac{C F(\text { year })-C F(2007)}{C F(2007)} \times 100
$$

Table 7: Comparison of CFs between year 2007 and other years.

\begin{tabular}{|c|c|c|c|c|}
\hline \multirow{2}{*}{ Energy } & \multicolumn{5}{|c|}{ Percentage deviation $(\Delta \%)$} \\
\cline { 2 - 5 } & $\mathbf{2 0 1 0}$ & $\mathbf{2 0 1 1}$ & $\mathbf{2 0 1 2}$ & $\mathbf{2 0 1 3}$ \\
\hline 47 & 7.39 & 13.83 & 18.96 & -40.52 \\
\hline 84 & 6.93 & 15 & 16.14 & -35.87 \\
\hline 121 & 7.11 & 12.66 & 14.62 & -24.86 \\
\hline 171 & 7.70 & 15.57 & 19.74 & -17.90 \\
\hline 218 & 5.24 & 12.06 & 20.52 & -21.06 \\
\hline 662 & 7.21 & 11.73 & 13.99 & -17.20 \\
\hline 1250 & 2.04 & 4.01 & 8.74 & -19.39 \\
\hline
\end{tabular}

*The acronyms in Tables $(2,3,4,5$, and 6$)$ are explained in Table 8.

Table 8: The acronyms in Tables (2, 3, 4, 5, and 6).

\begin{tabular}{|c|c|c|c|c|c|}
\hline & Acronyms & Meaning & & Acronyms & Meaning \\
\hline 1. & R.Q & Radiation Quality & 8. & CF & Calibration Factor \\
\hline 2. & $\mathrm{C} \mathrm{mA}$ & Current & 9. & $\mathrm{R}(1 / \mathrm{CF})$ & Response \\
\hline 3. & $\mathrm{E} \mathrm{KeV}$ & Energy & 10. & $\mathrm{~T}$ & Temperature \\
\hline 4. & E.C nC/min & Electrometer Charge & 11. & $\mathrm{P}$ & Pressure \\
\hline 5. & S.R mSv/y & Standard Reading & 12. & $\mathrm{H}$ & Humidity \\
\hline 6. & SM.R mSv/y & Survey Meter Reading & & & \\
\hline 7. & CF.S mSv/nC & Calibration Factor Standard & & & \\
\hline
\end{tabular}

Radiation survey meters are used to measure the presence of any radiation from radioactive sources or radiation generators such as X-ray machine and linear accelerator. The proper calibration of these instruments is to ensure that all meters are working correctly. Annual calibrations are required for all the type of survey meters. But the survey meters use in the radiation facilities with very high activity sources (eg: Co-60 plant), the calibration should be done at once in six months. Portable radiation survey meters must be calibrated at least annually to an accuracy of \pm 20 percent for the gamma energy of the sources in use. Portable radiation survey meters must be of a type that does not saturate and read zero at high radiation dose rates.

The calibration of ion chamber is mandatory to fulfill the legal requirement and IAEA recommendation to ensure that the measurements are compatible with those made elsewhere, assure constancy to promote customer acceptance. The pressurize ionization chamber was calibrated on 06 Sep 2013, with Co-60, Cs-137 gamma energies and five energies of $x$ rays.

The calibration factors of ionization chamber survey meter are changing from time to time.

For example, the calibration factors of survey meter are deviated from $-41 \%$ to $+21 \%$ compare with calibration factor of 2007 (refer Table.7).

The ionization chambers are sensitive, reliable and good energy response to $\mathrm{x}$ ray and gamma radiation. They should be maintained and keep carefully in the dry cabinet if they aren't in use.

The poor maintenance will give high background reading of the survey 
meter due to leakage current. High humidity can cause the massive variation in $\mathrm{CF}$. The chamber is very sensitive to the humidity which can cause high leakage current of the chamber and that leads to high variation of $\mathrm{CF}$. To reduce the avoidable leakage due to humidity, chamber must be kept inside a dry cabinet or desiccator which might be kept relative humidity always less than $50 \%$.

The insulator of the chamber must be with low surface conductivity to reduce the unwanted component to the signal through leakage as well. The resistance of the insulator must be greater than $1016 \Omega$. Thus the instrument should be compiled with the insulators as amber, polystyrene, polyethylene, nylon and Teflon.

When Calibrate the survey meters,

- Calibrations are to be conducted in an isolated area of the facility where the background radiation is low.

- The individual conducting the calibration shall wear a dosimetry badge.

- A calibrated survey meter should be used as a referenced standard to ensure that unexpected changes in exposure rates are identified.

- A radioactive sealed point source shall contain a nuclide which emits a strong enough radiation field of similar type and energy that would be seen in labs.

- The survey meter calibration should be considered successful if the exposure rate differs from the calculated rate by less than $20 \%$.

- Records shall be kept of each survey performed, and will include owner of instrument; manufacturer's name, model number and serial number; signature of individual who performed the calibration; date the calibration was performed; and next expected calibration date.
- All records of calibration results should be kept for at least three years or requirement of the organization. Radiation Safety Officer should be informed, if any survey meter calibration is out of date or any new purchase or disposal of survey meters.

The results can be concluded that the ionization chamber or survey meters must calibrate annually and must maintain carefully to reduce the discarded effects to the measurements. The calibration of survey meter should be performed by means of gamma sources such as Cs-137 and Co-60 and $\mathrm{X}$-rays.

\section{References}

[1] International Commission on Radiation Units and Measurements. Determination of Dose Equivalents Resulting from External Radiation Sources. ICRU Report 39, 1985.

[2] International organization for standardization, $\mathrm{X}$ and Gamma Reference Radiation for Calibrating Dose Meters and Dose Rate Meters and for Determining Their Response as a Function of Photon Energy Characteristics of The Radiations and Their Methods of Production, ISO Standard 4037-1, Geneva (1995).

[3] International Atomic Energy Agency. Calibration of Radiation Protection Monitoring Instruments. Safety Reports Series No. 16, IAEA, Vienna, 2000.

[4] International Atomic Energy Agency. Radiation Oncology Physics: A Handbook For Teachers And Students, IAEA, Vienna, 2005.

[5] International Atomic Energy Agency, Calibration of Radiation Protection Monitoring Instruments. Safety Reports Series No. 16, IAEA, Vienna, 1971.

[6] International Atomic Energy Agency, The Safe Use of Radiation Sources, Training Course Series No. 6, IAEA, Vienna (1995). 
[7] N. M. Ridler and M. J. Salter, "An approach to the treatment of uncertainty in complex S-parameter measurements" Metrologia, 39 (2002) 295-302.

[8] McEwen MR, Williams AJ, DuSautoy AR. Determination of absorbed dose calibration factors for therapy level electron beam ionization chambers. Phys Med Biol 46 (2001) 741-755. 